\title{
Empirical Probability Based QoS Routing
}

\author{
Xin Yuan Guang Yang \\ Department of Computer Science, Florida State University, Tallahassee, FL 32306 \\ \{xyuan,guanyang\}@cs.fsu.edu
}

\begin{abstract}
-
We study Quality-of-Service $(\mathrm{QoS})$ routing schemes that make routing decisions based on empirical resource availability probability information. These empirical probability based routing schemes offer better performance than traditional schemes that make routing decisions based on resource availability information when the global network state information is imprecise. We investigate variations of empirical probability based QoS routing, present a number of schemes to explicitly maintain the resource availability probability information, and evaluate the performance of the routing schemes. We conclude that the performance of empirical probability based routing is insensitive to the frequency of probability information updates and that empirical probability based routing can achieve good performance without introducing excessive overheads.
\end{abstract}

\section{INTRODUCTION}

Traditionally, Quality-of-Service (QoS) routing uses the network resource availability information to make routing decisions. We will call such algorithms availability based QoS routing algorithms. One of the major problems in availability based QoS routing is that the global network state information is usually inaccurate. The inaccuracy may be introduced by many factors such as non-negligible propagation delay and hierarchical topology aggregation [6]. The inaccurate global network state information can greatly affect the performance of the routing algorithms [1], [9].

An alternative to availability based routing is probability based routing [1], [4], [5], [6]. In probability based routing, the resource availability probability information is either maintained or inferred, and the routing decision is made based on the probability information. Probability based routing potentially offers the solutions for the problems caused by the imprecise network state information since the resource availability probability distribution for a link changes much slower than the resource availability of the link. Although probability based routing has been proposed, the feasibility and effectiveness of this method has yet to be thoroughly investigated and many questions remain to be answered: How to obtain the resource availability probability information? What is the overhead in the maintenance of resource availability probability information? How to use of the probability information to make effective routing decisions? What are the strengths and weaknesses of probability based routing in comparison to traditional availability based routing? In this paper, we attempt to answer some of these questions.
We consider bandwidth-constrained problems in the context of source routing. We will assume that a link state algorithm that periodically updates the state information is used to maintain the global network state information. We propose to explicitly maintain the empirical bandwidth availability probability information using the link state algorithm. We investigate various methods to obtain empirical bandwidth availability probability information and to compute feasible paths using both the probability information and the bandwidth availability information, and perform extensive simulation to evaluate the routing algorithms. The main conclusion of this study include the followings:

- The performance of the empirical probability routing algorithms is insensitive to the frequency of the probability information updates. Thus, empirical probability based QoS routing is feasible and can achieve good performance without introducing excessive overheads although maintaining the probability information requires more data to be exchanged than maintaining the availability information.

- Empirical probability based routing is better than traditional availability based routing only when the global state information is imprecise. When the global network state information is sufficiently accurate, availability based routing offers better performance.

- For empirical probability based routing, different methods to obtain the probability information result in different performance. A naive empirical probability based routing method does not yield the best performance.

The rest of the paper is organized as follows. In Section 2, we describe the related work. In Section 3, we introduce various empirical probability based routing schemes. In Section 4 , we present the results of the performance study. Section 5 concludes the paper.

\section{RELATED WORK}

QoS routing has attracted much attention recently. A recent survey can be found in [3]. One of the major issues in QoS routing is dealing with the imprecise global network state information. A number of QoS routing schemes were proposed to deal with the imprecise state information [1], [2], [4], [5], [6], [8]. A comparative study of these routing schemes is presented in [11]. Probability based routing [1], [4], [5], [6] is one of the schemes that deal with imprecise state information. It was first introduced in [5], [6] where routing 
algorithms for bandwidth-constrained and delay-constrained problems were developed and a number of important theoretical results for probability based routing were obtained. These papers, however, do not discuss how to obtain the probability information. In [1], the probability based QoS routing scheme in [5] was materialized to be the safety-based routing where the link bandwidth availability probability is inferred from the link state information by considering the details about the link state update policy. The technique does not require the maintenance of the empirical probability information and it only works for certain link state update policies. An empirical probability based approach is proposed for hierarchical QoS routing [4]. In this paper, we develop new schemes that result in more accurate probability information and higher routing performance than the technique in [4].

\section{EMPIRICAL PROBABILITY BASED QOS ROUTING}

Empirical probability based QoS routing for bandwidthconstrained problems consists of two parts, the maintenance of the probability information and the path computation using the probability information. This work focuses on the maintenance of the probability information. We assume that the shortest-safest routing (ssr) algorithm [1] is used to compute feasible paths. The $s s r$ algorithm first determines all paths that have the largest probability to satisfy a connection request, and then selects the shortest one for the connection request. For a connection that requires $x$ units of data, $s s r$ uses the knowledge of the probability $p_{l}(x)$ that link $l$ can support the connection to compute feasible paths. This probability information for all links in the network is to be obtained and maintained by a probability information maintenance scheme. Next, we will describe methods to obtain and maintain the empirical probability information.

\section{A. Availability independent probability distribution}

In this section, we will describe an approach to obtain the empirical availability independent probability information. This approach is similar to the method in [4].

The empirical bandwidth availability probability distribution of a link can obtained as follows. Let the total bandwidth for the link be bandwidth. The range of the bandwidth is partitioned into $n$ bandwidth levels: $0=b_{1}<b_{2}<\ldots<b_{n-1}<$ $b_{n}=$ bandwidth. A counter vector $\vec{v}=\left(v_{1}, v_{2}, \ldots, v_{n}\right)$ is associated with each link. The available bandwidth in each link is periodically sampled. When the sampled bandwidth, $b$, is in the range $\left[b_{i}, b_{i+1}\right)$, that is, $b_{i} \leq b<b_{i+1}$, the corresponding counter $v_{i}$ is incremented. The empirical probability mass function of the bandwidth availability can be derived from the counter vector. Let $\overrightarrow{v^{l}}=\left(v_{1}^{l}, v_{2}^{l}, \ldots, v_{n}^{l}\right)$ be the counter vector associated with link $l$. The probability that the bandwidth on link $l, b w$, falls in the range $\left[b_{i}, b_{i+1}\right)$ is

$$
P\left(b w \in\left[b_{i}, b_{i+1}\right)\right)=\frac{v_{i}^{l}}{\sum_{i=1}^{n} v_{i}^{l}},
$$

and the probability that the link $l$ can support a connection with $x$ units of bandwidth, $p_{l}(x)$, can be approximated as

$$
p_{l}(x)=\frac{\sum_{b_{i} \geq x} v_{i}^{l}}{\sum_{i=1}^{n} v_{i}^{l}} .
$$

By distributing the counter vector for a link using the link state algorithm, the empirical bandwidth availability probability information can be obtained by all the nodes in a network. Maintaining the probability information incurs larger overheads than maintaining the availability information. Exchanging the counter vector requires $O(n)$ data to be distributed, where $n$ is the number of bandwidth levels for the link, while exchanging bandwidth availability only needs to distribute $O(1)$ data. The frequency for the probability information updates does not need to be the same as the frequency to distribute the bandwidth information. Since we assume periodical link state updates in this paper, we will distinguish the two update frequencies and use link state update interval to denote the interval between two consecutive bandwidth availability updates and probability update interval to denote the interval between two consecutive probability information updates. We will call QoS routing with the availability independent probability information basic probability based routing.

\section{B. Availability dependent empirical probability distribution}

One problem with the basic probability based routing scheme is that the probability information may not be accurate. Intuitively, a link with an advertised bandwidth of $100 \mathrm{Mbps}$ is more likely to be able to support a connection that requires $90 \mathrm{Mbps}$ bandwidth than a link with an advertised bandwidth of $1 M b p s$. Since the link state information is usually available, we should seek methods to use this information to improve routing performance.

One way to use the advertised bandwidth availability information is to cut-off links that currently have below threshold bandwidths. To find a path that requires $x$ units of bandwidth, the links whose current advertised bandwidths are less than $x$ units of bandwidth are not considered in the routing process. The probability based routing will be performed in the rest of the graph. We will call such a scheme the basic plus or plus probability based scheme.

Another way is to maintain empirical time-availability dependent conditional probability information, which allows more accurate probability information to be derived. The idea is to maintain the following conditional probability: after a link is advertised as having $x$ units of available bandwidth at time $t$, the probability that the link will have $y$ units of available bandwidth at time $t+\Delta t$. When $\Delta t$ is approaching infinity, the probability distribution should approach the availability independent probability. Thus, we can have a time $T$ such that when $\Delta t>T$, the bandwidth availability probability distribution is approximated by the availability independent probability distribution. The time range $[0, \mathrm{~T}]$ is partitioned into $m$ 
steps, $0=t_{1}<t_{2}<\ldots<t_{m}=T$. Let the bandwidth in a link bandwidth be partitioned into n levels: $0=b_{1}<b_{2}<$ $\ldots<b_{n-1}<b_{n}=$ bandwidth. The empirical conditional probability distribution for a link $l$ can be maintained using $n \times m$ vectors, $\overrightarrow{v^{l}}(1 . . n, 1 . . m)$, of counters with each vector of counters $\overrightarrow{v^{l}}(i, j)=\left(v_{1}^{l}(i, j), v_{2}^{l}(i, j), \ldots, v_{n}^{l}(i, j)\right)$, where $1 \leq i \leq n$ and $1 \leq j \leq m$, recording the empirical conditional probability for time $t_{j}$ after the link declares to have a bandwidth in the range $\left[b_{i}, b_{i+1}\right)$. In addition to the periodic sampling for the availability independent probability, the router that is connected to link $l$ also samples the available bandwidth on link $l$ at time $t_{1}, t_{2}, \ldots t_{m}$ after a bandwidth availability advertisement for link $l$ is sent out. If the sampled bandwidth is $s b, b_{j} \leq s b<b_{j+1}$ at time $t_{k}$ after the available bandwidth $a b, b_{i} \leq a b<b_{i+1}$, is advertised, the counter $v_{j}^{l}(i, k)$ is incremented. Note that for a link state algorithm with periodic link state updates, if the link state update interval is less than $T$, we do not need to approximate using the availability independent probability.

The $n \times m$ vectors of counters essentially record the conditional probability for times $t_{1}, t_{2}, \ldots, t_{m}$ after a link advertises its bandwidth. Let the last link state advertisement for link $l$ to be $b$ units, where $b_{i} \leq b<b_{i+1}$. At time $t, t_{k} \leq t<t_{k+1}$, after the last link state update, the probability that the link bandwidth on link $l, b w$, falls into the range $b_{j} \leq b w<b_{j+1}$ can be calculated as

$$
P\left(b w \in\left[b_{j}, b_{j+1}\right)\right)=\frac{v_{j}^{l}(i, k)}{\sum_{j=1}^{n} v_{j}^{l}(i, k)},
$$

and the probability that the link $l$ can support a connection with $x$ units of bandwidth requirement at this time, $p_{l}(x)$, can be approximated as

$$
p_{l}(x)=\frac{\sum_{b_{j} \geq x} v_{j}^{l}(i, k)}{\sum_{j=1}^{n} v_{j}^{l}(i, k)} .
$$

Notice that the calculation of the probability that a link can support a given bandwidth is no more complex than that in the basic method, although much more information is to be distributed to obtain the conditional probability information. Thus, using conditional probability does not increase the complexity of path calculation. Notice also that maintaining this conditional probability for a link requires $O\left(n^{2} m\right)$ data $(n \times m$ vectors of size $n$ ) to be distributed, where $n$ is the number of bandwidth levels and $m$ is the number of time steps. However, as demonstrated in the performance study, the probability distribution information can be exchanged infrequently without significantly affecting the routing performance. It is, thus, possible to maintain this time-availability dependent probability information without incurring excessive overheads. We will call this approach timed probability based routing. Comparing to the basic method, the timed method introduces larger overheads to maintain more accurate probability information.

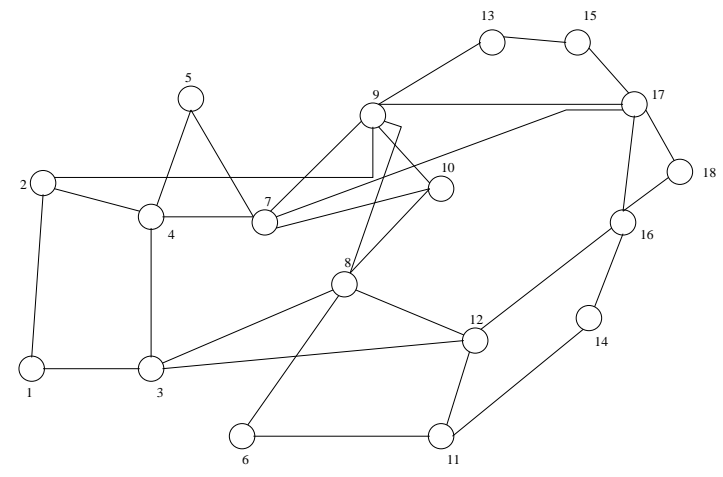

Fig. 1. The ISP topology

\section{PERFormanCE STUdy}

We carried out extensive simulation on a number of random topologies generated using Georgia Tech Internetwork Topology Models (GT-ITM) [12] and the ISP topology shown in Fig. 1. The random topologies studied include a number of 30-40 nodes topologies with an average nodal degrees of 3 and 4 . The trend in the results for these random topologies is similar to that for the ISP topology. We will only present the results for the ISP topology shown in Fig. 1.

All the links are assumed to be bi-directional and of the same capacity, with $C$ units of bandwidth in each direction. Flows arrive at a node according to a Poisson process with a rate $\lambda$. The destination node is chosen randomly from all nodes except the source node. The connection holding time is exponentially distributed with a mean of $1 / \mu$ seconds. The bandwidth requirement of the flows is exponentially distributed with a mean of $B$ units. The offered network load is given by $\rho=\lambda N h^{\prime} B / \mu L C$, where $N$ is the number of nodes, $L$ is the number of links, $h^{\prime}$ is the mean number of hops per flow, averaged across all source-destination pairs. The parameters used in this simulation are $C=100, N=18, L=60$. $h^{\prime}=2.36$. Two traffic patterns are used in the experiments: long duration with small bandwidth requirement and short duration with large bandwidth requirement. The long duration with small bandwidth requirement traffic, denoted as the $(5-60)$ traffic, consists of flows with $B=5$ and $1 / \mu=60$. The short duration with large bandwidth requirement traffic, denoted as the (20-15) traffic, consists of flows with $B=20$ and $1 / \mu=15$. The $(5,60)$ traffic results in more accurate link state information than the $(20,15)$ traffic.

We study the three empirical probability based methods discussed in this paper, the basic method, the plus method, and the timed method. For comparison, we also include the results for the traditional availability based routing method, widestshortest routing (wsr) [7]. The wsr algorithm finds the shortest path that can satisfy the connection requirement. When there are multiple shortest paths available, $w s r$ selects the one that has the largest available bandwidth for the connection. For all the probability methods, the link bandwidth (100 units) is partitioned into 100 levels. For the timed probability based 
routing with a link state update interval of $I$ seconds, the time interval $[0, I)$ is partition into $I$ time steps with 1 second in each step. A blocked flow is dropped without being retried. All the results are obtained with a $99 \%$ confidence level.

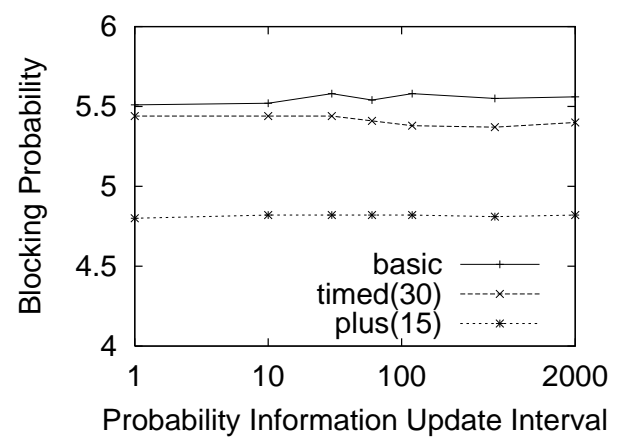

Fig. 2. The impact of the probability information update interval

One of the most important issues to be considered is the overheads in the probability based routing. Two factors contribute to the overheads: the size of link state advertisement (LSA) packets and the frequency to distribute the LSAs. As discussed earlier, maintaining the link bandwidth probability information requires larger LSAs. This experiment focuses on the probability information update frequency. Fig. 2 shows the impact of the probability information update interval. In this experiment, the offer load is 0.7 with the $(5-60)$ traffic. In the figure (and throughout the rest of this paper), we will use notion method $(I)$ as the legend for the results for the particular method with a link state update interval of $I$ seconds. For example, plus(15) means the plus method with a link state update interval of 15 seconds. As can be seen from the figure, all probability based methods are quite insensitive to the probability information update interval. Updating the probability information every 2000 seconds yields almost the same performance as updating the probability information every second. The same results hold for other settings with different traffic patterns, different topologies, and different network loads. The empirical probability is basically calculated from the history of link usages. As long as the traffic pattern does not change, the probability information will become more accurate as more history information is accumulated regardless of the update interval. This experiment indicates that empirical probability based routing can be performed with infrequent probability information updates without losing performance and the infrequent updates justify the larger LSAs. Since the probability update interval does not significantly affect the performance, in the rest of the paper, a probability update interval of 120 seconds is used in all the results for all probability based routing methods.

Fig. 3 and 4 show the impact of the link state update interval. Fig. 3 shows the results for the $(5-60)$ traffic and Fig. 4 shows the results for the $(20-15)$ traffic. The network load is 0.7 . For both traffic patterns, the timed approach

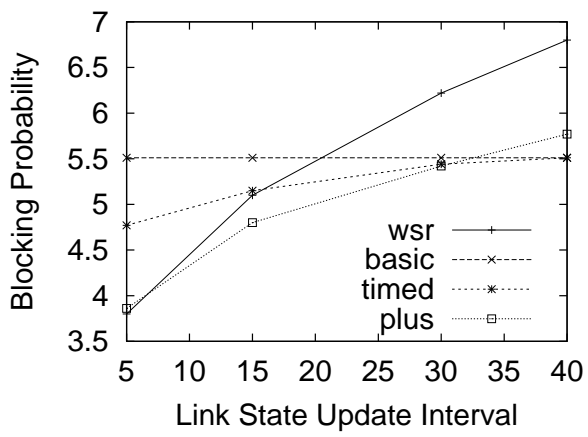

Fig. 3. Impact of the link state update interval for $(5-60)$ traffi c

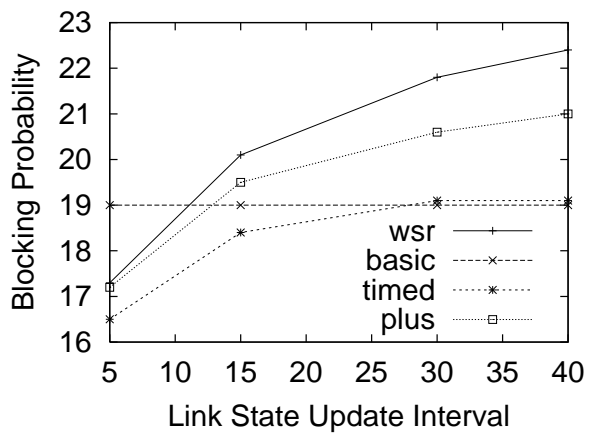

Fig. 4. Impact of the link state update interval for $(20-15)$ traffi c

consistently offers good performance compared to the basic approach. Among the probability based schemes, the plus approach is most sensitive to the link state update interval. It yields better performance than the basic method when the link state information is sufficiently accurate and worse performance when the link state information is sufficiently inaccurate. All the probability based methods are less sensitive to the link state update interval than the availability based routing wsr. However, when the link state information is very accurate, as in the case for the $(5-60)$ traffic with a link state update interval of 5 seconds, wsr is better than all the probability based methods. This is mainly due to the better path computation heuristic used in $w s r$ since $w s r$ biases towards short paths and take the load balancing factor into consideration in the path computation process. Thus, whether availability based routing or probability based routing would have a better performance depends on the traffic pattern and the accuracy of the global state information.

Fig. 5 and 6 show the performance of routing algorithms with different network loads. These experiments assume that the link state update interval is 15 seconds. For the $(5-60)$ traffic, wsr performs better than the basic method when the network load is low. As the network load becomes higher, the state information becomes imprecise and the performance of $w s r$ decreases as compared to the probability based methods. In this setting where the network state information is still fairly accurate, the plus method results in the best performance 


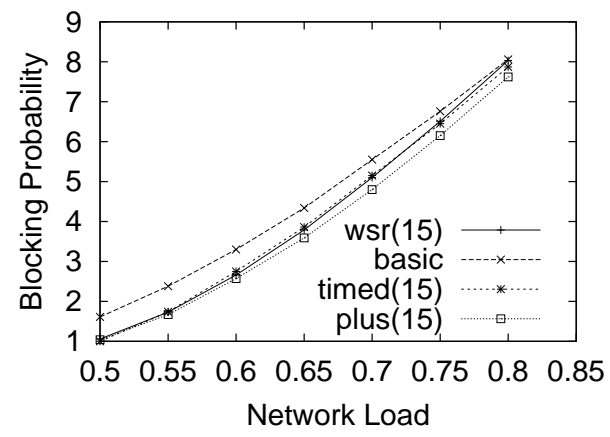

Fig. 5. Performance for $(5-60)$ traffi c

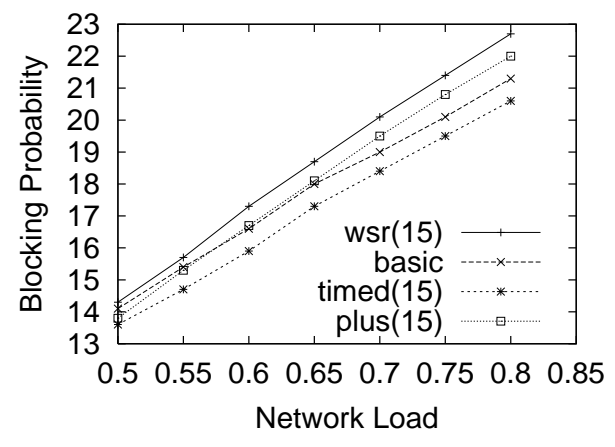

Fig. 6. Performance for $(20-15)$ traffi c

among all the schemes. For the $(20-15)$ traffic, the link state information is more inaccurate and the probability based approaches all perform better than $w s r$ for all network loads. In this setting, the timed method is the best. Combining these results with the results in previous experiments, we can see that when the state information is inaccurate, the pure probability based methods, such as the basic and the timed methods, perform better. When the state information is more accurate, the plus scheme can achieve good performance. When the state information is very accurate, availability based routing, wsr, can be the best among all the schemes studied.

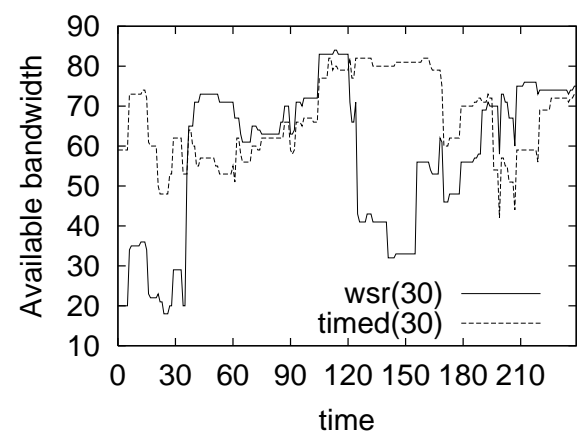

Fig. 7. Snapshots of the bandwidth availability on link $1 \rightarrow 3$

Fig. 7 shows snapshots of the bandwidth availability on link $1 \rightarrow 3$ for $w s r$ and timed probability based routing with a 30 seconds link state update interval. This experiment assumes a network load of 0.6 and the $(5-60)$ traffic. The snapshot is taken for the virtual time from 100020 seconds to 100260 seconds in the simulation. From the figure, it is clear that $w s r$ suffers from bandwidth oscillation which affects the routing performance. The bandwidth usage in the the timed probability based routing scheme is much smoother. This is one reason why the probability based routing is attractive when the global network state information is imprecise.

\section{CONCLUSION}

In this paper, we propose to explicitly maintain the empirical link bandwidth probability information through the link state algorithm and investigate variations of empirical probability based QoS routing. We find that the performance of the empirical probability based routing is insensitive to the frequency of probability information updates, which indicates that empirical probability based routing is feasible even though it requires a larger amount of data to be exchanged. We further study different methods to obtain and use the probability information and conclude that more accurate empirical probability information and better routing results can be obtained by maintaining the time-availability dependent conditional probability.

\section{REFERENCES}

[1] G. Apostolopoulos, R. Guerin, S. Kamat, and S. Tripathi, "Improving QoS Routing Performance Under Inaccurate Link State Information." Proceedings of the 16th International Teletraffic Congress, June 7-11, 1999.

[2] S. Chen and K. Nahrstedt, "Distributed QoS Routing with Imprecise State Unformation.” ICCCN'98, October 1998.

[3] S. Chen and K. Nahrstedt, "An Overview of Quality-of-Service Routing for the Next Generation High-Speed Networks: Problems and Solutions," IEEE Networks, Special Issue on Transmission and Distribution of Digital Video, Nov./Dec., 1998.

[4] D. Ghosh and R. Acharya, "A Probabilistic Approach to Hierarchical QoS Routing", IEEE International Conference on Computer Networks, October, 2001.

[5] R. Guerin and A. Orda, "QoS-based Routing in Networks with Inaccurate Information: Theory and Algorithms.”, IEEE INFOCOM'97, April 1997.

[6] Dean H. Lorenz and Ariel Orda, "QoS Routing in Networks with Uncertain Parameters", IEEE/ACM Transactions on Networking, Vol. 6, No. 6, pages 768-778, December 1998.

[7] Q. Ma and P. Steenkiste, "Quality-of-Service Routing with Performance Guarantees." 4th International IFIP Workshop on Quality of Service, May 1997.

[8] Srihari Nelakuditi, Zhi-Li Zhang, and Rose P. Tsang, "Adaptive Proportional Routing: A Localized QoS Routing Approach", In IEEE Infocom, April 2000.

[9] A. Shaikh, J. Rexford and K. Shin, "Evaluating the Overheads of Source-Directed Quality-of Service Routing", International Conference on Network Protocols (ICNP), 1998.

[10] X. Yuan and X. Liu, "Heuristic Algorithms for Multi-Constrained Quality of Service Routing", IEEE INFOCOM 2001, pages 844-853, Anchorage, Alaska, April 22-26, 2001.

[11] X. Yuan, W. Zheng and S. Ding, "A Comparative Study of Quality of Service Routing Schemes That Tolerate Imprecise State Information", the 11th IEEE International Conference on Computer Communications and Networks (IC3N'02), Miami, FL, October, 14-16, 2002.

[12] Ellen W. Zegura, Ken Calvert and S. Bhattacharjee. "How to Model an Internetwork." Proceedings of IEEE Infocom '96, 1996. 